\title{
Storytelling in research
}

\author{
The communication of research findings, in both written and oral forms, would benefit from concepts used in \\ cinematographic storytelling.
}

R aw data alone does not make a story. Neither does a rigorous yet plain description of the data. Raw data and purely descriptive text are particularly useful when they have to be mined by computers, but they do not effectively communicate research for others to interpret, learn or build on. Even specialists on the topic may need guidance on the interpretation of the data and on the overall purpose of the experiments (or simulations or theory). Experts also appreciate a narrative that exposes the background and rationale of the work, as well as its broad relevance and implications.

Yet many scientists and the public consider that scientific papers, and even oral communication of research, should be presented in a wholly objective and dry form, and that such reports should avoid any discussion of the authors' motives for the research and their interpretation of its outcomes. This is bad advice. Data should indeed be objective and informative, and thereby provide credibility; yet the way the data are constructed, presented and delivered matters. An engaging narrative that explains the findings, provides context and assists interpretation while avoiding hype can greatly enhance the reach of the work beyond the typically few specialists who can readily make sense of the naked data.

Take, for instance, a report on results from an improved liquid-biopsy assay. In addition to the necessary data about the performance of the test (such as the cumulative distribution functions of sensitivity and specificity of the assay and the proportion of cancer-positive patients) and the clinical characteristics of the patients, a compelling narrative would highlight the need for the particular assay, its advantages and limitations with respect to other liquid-biopsy tests, how the assay was designed, the trade-offs in its design, materials, applicability and usability, and the roadblocks that would have to be overcome for eventual implementation in clinical or field settings. Far from being truly objective, these aspects put the authors at the centre of the story. Research is a human endeavour, and hence the communication of research findings shouldn't exclude the researchers' motivations, analysis and vision.

How best to convey those more personal aspects? Everyone enjoys stories, and we

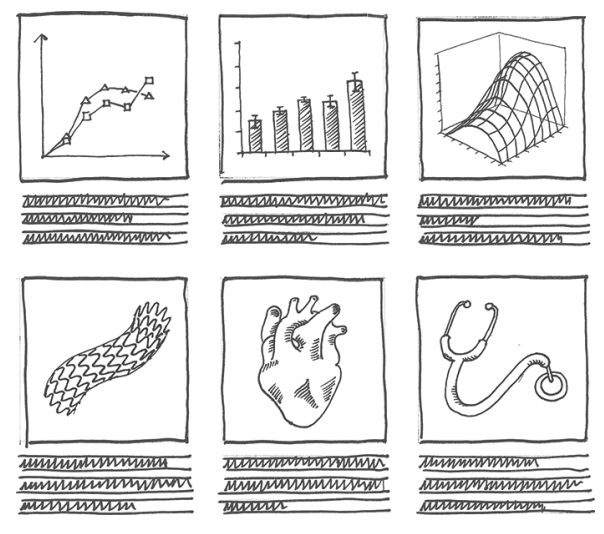

learn and retain facts best when the stories that wrap the facts sustain the reader's attention. In that regard, cinematography is particularly successful, and not only because the combination of dynamic imagery and sound can be more engaging than static text and figures on a page. Cinematographic storytelling also makes good use of storyboards, composition, framing, camera movements and lighting. These concepts can be applied to storytelling in research, such as the writing of a research grant, paper or journalistic piece, and the creation and delivery of an oral presentation.

For example, the outline of a paper can be the analogue of the storyboard in film-making. As with movies, a strict chronological account of the findings (in a movie, the action) is most often not the best way to tell the story. Also, the main figures and findings (the main plot) and the secondary details (subplots) can be arranged in the outline in a way that is most logical and best facilitates comprehension. However, whereas the main purpose of cinematography is to entertain, scientific papers aim to explain. Hence, in scientific storytelling, the punch line should not be left to the end, and hinting at or hiding key elements should not be used as a way to keep the reader's attention. Mystery, twists and surprises should be left aside. When writing papers and giving presentations, the central points should be stated early and clearly, and the context, findings and implications must be provided, in that order. The storyboard analogy can, however, help when deciding the logical flow of the description of the results, how they relate to each other (especially when there are parallel threads to the main story), and their context and implications.

Composition and framing can also be applied, both to figures and to the text. Graphs that need more attention (because of complexity or difficulty of interpretation, for example) can be made more visually prominent, and related data laid out so as to indicate logical relationships. Multi-panel figures should be composed to best guide the reader through the data, in concordance with the story (for example, it is easier for the eye to compare data in bar graphs when they appear side-by-side than when they are stacked). And the study should be framed to convey the motivation and provide the most appropriate context. In particular, it should be clear from the introduction whether the purpose of the study is to test a hypothesis, to validate previous results, to improve known outcomes or to provide new methodology. Context can be narrated by starting with a broad perspective of the field (in cinematography, an extreme wide shot) and then by gradually focusing on the main purpose of the study (the central character of the plot), describing what is known and the gaps in knowledge (wide and medium long shots). The description of sets of results can combine specific details (extreme close-up shots) and summaries (head-and-shoulder shots).

Applying the concepts of camera motion and lighting to narrative can help the writer or speaker transition between related results and discussion, and can be used to emphasize primary points. For example, a paragraph or slideshow can 'pan' through a range of complementary methods, 'lighting up' the critical aspects of each one, before a 'scene shift' introduces a new approach. Zooming into specific aspects of the approach and temporarily stopping the discussion flow to describe a particular point from multiple angles (akin to the bullet-time visual effects in that famous scene in The Matrix) could help the reader better grasp the advantages of the approach.

As an author, you are the director of your own research story. Make it engaging.

Published online: 6 February 2018 https://doi.org/10.1038/s41551-018-0202-5 